\title{
Different Approaches to Coordinate Transformation Parameters Determination of Nonhomogeneous Coordinate Systems
}

\author{
Roman Shults (D) ${ }^{*}$, Asset Urazaliev ${ }^{2}$, Andriy Annenkov ${ }^{3}$, Olena Nesterenko ${ }^{4}$, \\ Oksana Kucherenko ${ }^{5}$, Kateryna Kim ${ }^{6}$ \\ 1, 3, 5 Department of Engineering Geodesy, School of GIS and Land Management, \\ Kyiv National University of Construction and Architecture, Kyiv, Ukraine \\ ${ }^{2}$ Department of Cartography and Geoinformatics, Faculty of Geography and \\ Environmental Sciences, Al-Farabi Kazakh National University, Almaty, Kazakhstan \\ ${ }^{4,6}$ Department of GIS and Photogrammetry, School of GIS and Land Management, \\ Kyiv National University of Construction and Architecture, Kyiv, Ukraine
}

Received 04 February 2020; accepted 31 March 2020

\begin{abstract}
During reconstruction and restoration of city geodetic networks, there is quite a common problem that is related to the nonhomogeneity of existing geodetic networks. In any city, local authorities operate with their coordinate systems. Such conditions lead to inconsistency between data of different services. There is only one way how to overcome the problem that lies in the creation and deployment of the new common coordinate system for the whole city. But such an approach has a lack connected with the necessity of transformation parameters acquisition for the latest and old coordinate systems. Insofar as old coordinate systems had been created with different accuracy, using various equipment, and measuring technologies, it is not possible to consider them as homogeneous. It means that we cannot use a classical conformal Helmert transformation to link different coordinate systems. In the presented paper were studied the different approaches for transformation parameters acquisition. A case study of the Almaty city coordinate system was researched and compared the following methods: Helmert transformation, bilinear transformation, the second and third-order regression transformation, and the fourth-order conformal polynomial transformation. It was found out that neither of the considered methods maintains the necessary transformation accuracy $(>5 \mathrm{~cm})$. That is why the creation of the transformation field using the finite element method (FEM) was suggested. The whole city was divided into triangles using Delaunay triangulation. For each triangle, the transformation parameters were found using affine transformation with the necessary accuracy.
\end{abstract}

Keywords: coordinate system, nonhomogeneity, geodetic network, polynomial transformation, affine transformation, finite element method.

\section{Introduction}

City geodetic networks are designed to provide large-scale mapping, in which the relief of the terrain and infrastructure elements are displayed with minimal distortion. This task is achieved by establishing and using a local 2D planar rectangular coordinate system. Also, for obvious reasons, the coordinate system adopted to create maps should remain stable. Today, it is advisable to create modern city geodetic systems using satellite technologies. Using GNSS equipment, the coordinates are being determined in the global geodetic coordinate system WGS84 with a transformation to UTM coordinates. For the GNSS receiver to work in the local coordinate system, we need to specify this coordinate system (CS) in the receiver. It is possible to select such a system either in the list of supported CSs or manually set transformation parameters - a transformation key that describes how the local system relates to WGS84. The search for that key has serious difficulties. The determination of the key is carried out by comparison of reference points in the local CS and global CS. When searching for the key to the local coordinate system, it is essential to find a direct transition from the planar local CS and UTM that is a derivative of the WGS84 coordinate system.

At the moment, almost all the geodetic networks on the territory of Kazakhstan were created and are being created on a mathematical basis that is different from used for other countries. In particular, Kazakhstan geodetic networks use the systems SK-42 and SK-63, which are based on the Krassovskiy ellipsoid. These geodetic networks are nonhomogeneous, being that measurements were done by various equipment and processed with different algorithms.

${ }^{*}$ Corresponding author. E-mail: shultsrv@ gmail.com 
In any city, local authorities operate with their local 2D coordinate systems. Such conditions lead to data inconsistency. It is certainly valid for the biggest city Almaty. For such a case, the use of a conventional transformation, known as Helmert transformation, is hardly possible. That is why it is necessary to consider another way of the transformation parameters search. There are many well-known approaches on how to overcome the problem of non-homogeneity during transformation. It is worth to mention the original work (Mikhail, 1976), where one may find expressions for Helmert transformation, affine transformation, polynomial transformation, and conformal polynomial transformation. A comprehensive description of those methods can be found in (Deakin, 2004; Gao, 2017; The International Association of Oil \& Gas Producers [IOGP], 2018). Since that time, little has been written about new methods. Thanks to deploying the high-productive PCs, today, it is possible to make use the complicated methods, such as FEM (Kohli \& Jenni, 2008) or neural networks (Gil \& Mrówczyñska, 2012; Ziggah et al., 2019).

The presented study is aimed at a comparison of different approaches to coordinate transformation parameters determination of nonhomogeneous coordinate systems. The sample for this study is the restoration and renovation of the Almaty local coordinate system. For that example, the effectiveness of those methods was evaluated.

This paper is divided into three sections. Section one provides a concise description of the Almaty local coordinate system and its restoration procedure. Section two details the transformation approaches, and section three outlines the results of the study.

\section{Object description}

On the territory of the city of Almaty, several types of coordinate systems are used. During the majority of geodetic works, the problem arises concerning the transformation of the received coordinates to one or another system. The latest measurements related to determining the coordinates of the local CS were performed on the territory of Almaty in 1998. Until now, a full survey of network points has not been carried out. All organizations conducting geodetic work in Almaty and nearby areas have used the coordinates of an outdated network. At the moment, cartographic documentation continues to be created in the system SK-63 or the local CS. The parameters of the transformation from one system to another are the following components: origin shift $\left(x_{0}, y_{0}\right)$; longitude of the meridian of the first zone $L_{0}$; the number of coordinate zone $N$; the angle of rotation of the coordinate axes $\theta$; the reference surface height $H_{0}$; scale factor. All of the above parameters are the key of the local CS. It is evident that for the goal of network restoration, such a set of parameters is cumbersome. Moreover, keeping in mind that the local CS is derivative from state CS, that is also obsolete and has serious flaws, there is no point in using the transformation key. The local deformations of the state CS distort the local CS and its transformation key. The city authority had accepted a decision about the new CS for Almaty to overcome this problem. Thus, to determine the distortions of the local CS of the city, it was decided to perform static GNSS measurements at the existing points of the geodetic network covering the entire territory of the city of Almaty. The scheme of the GNSS network is presented in Figure 1.

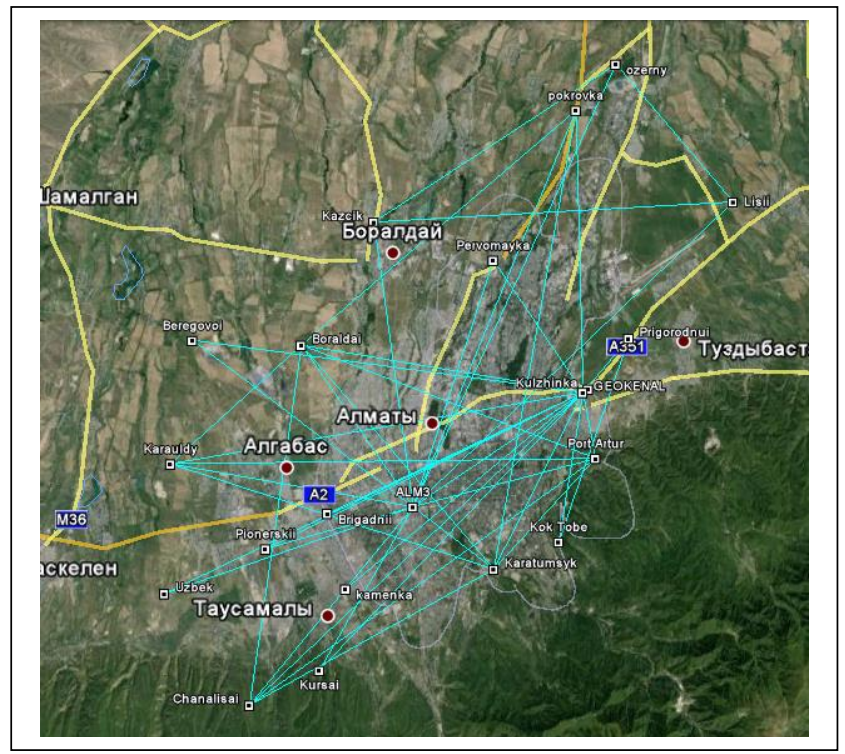

Figure 1. The scheme of the Almaty GNSS network

Therefore the 2D coordinates of the points have become known in the local CS and UTM. Despite that the coordinates in the new one system, namely UTM, were determined with considerably higher accuracy, the local authorities still demand the coordinates to be presented in both systems. That is why the problem of transformation parameters search is essential. However, the issue cannot be resolved conventionally, insofar as the deformations of 
the local CS have non-uniform distribution due to the reasons mentioned above and cannot be corrected by simple geometric transformations. Thus, different models to coordinate transformation parameters determination were studied.

\section{Transformation models}

The set of transformation models have been divided into two subsets: approximation models and interpolation models. Before we study and analyze the results of the research, it would be wise to detail different transformation strategies and mathematical models.

Let us commence with the approximation models. The transformation models below have been analyzed by order of complexity.

Helmert transformation. To find the transformation parameters, one uses the simplest model known as Helmert transformation.

$$
\begin{aligned}
& u=a_{1} x-b_{1} y+a_{0} \\
& v=b_{1} x+a_{1} y+b_{0} .
\end{aligned}
$$

This model allows describing the transformation through the four parameters: coordinates of origin, rotation angle, and scale.

Bilinear transformation. The more complicated is a model of bilinear transformation.

$$
\begin{aligned}
& u=a_{1} x+a_{2} y+a_{3} x y+a_{0} \\
& v=b_{1} x+b_{2} y+b_{3} x y+b_{0} .
\end{aligned}
$$

This model allows simulating simple local deformations.

Affine transformation. Among the classical geometric transformations in a plane, the most comprehensive is an affine transformation.

$$
\begin{aligned}
& u=a_{1} x+a_{2} y+a_{0} \\
& v=b_{1} x+b_{2} y+b_{0} .
\end{aligned}
$$

Polynomial transformation. Unlike the previous models that describe geometric transformations by the parameters that have geometric interpretation (shift, rotation, etc.), there is another case well-known as polynomial transformation. Among these transformations, the most popular is the second-order polynomial transformation

$$
\begin{aligned}
& u=a_{1} x+a_{2} y+a_{3} x y+a_{4} x^{2}+a_{5} y^{2}+a_{0} \\
& v=b_{1} x+b_{2} y+b_{3} x y+b_{4} x^{2}+b_{5} y^{2}+b_{0}
\end{aligned}
$$

and the third-order polynomial transformation

$$
\begin{aligned}
& u=a_{1} x+a_{2} y+a_{3} x y+a_{4} x^{2}+a_{5} y^{2}+a_{6} x^{3}+a_{7} x^{2} y+a_{8} x y^{2}+a_{9} y^{3}+a_{0} \\
& v=b_{1} x+b_{2} y+b_{3} x y+b_{4} x^{2}+b_{5} y^{2}+b_{6} x^{3}+b_{7} x^{2} y+b_{8} x y^{2}+b_{9} y^{3}+b_{0} .
\end{aligned}
$$

Polynomial conformal transformation It is well-known that parameters of polynomial transformation have no geometric meaning, and the transformation does not preserve shapes for infinitesimal regions. In different words, the polynomial transformation of orders higher than one violates the conformity condition. Polynomial conformal transformation is free of this lack, however, not for regions of finite size. We have used for our study the fourth-order conformal polynomial transformation:

$$
\begin{aligned}
& u=a_{1} x-a_{2} y+a_{3}\left(x^{2}-y^{2}\right)-a_{4} 2 x y+a_{5}\left(x^{3}-3 x y^{2}\right)-a_{6}\left(3 x^{2} y-y^{3}\right)+ \\
& +a_{7}\left(x^{4}-6 x^{2} y^{2}+y^{4}\right)-a_{8}\left(4 x^{3} y-4 x y^{3}\right)+a_{0} \\
& v=a_{2} x+a_{1} y+a_{4}\left(x^{2}-y^{2}\right)+a_{3} 2 x y+a_{6}\left(x^{3}-3 x y^{2}\right)+a_{5}\left(3 x^{2} y-y^{3}\right)+ \\
& +a_{8}\left(x^{4}-6 x^{2} y^{2}+y^{4}\right)+a_{7}\left(4 x^{3} y-4 x y^{3}\right)+a_{0} .
\end{aligned}
$$

For all the considered models, we may find the unknown parameters using standard least squares procedure that one can find in (Ghilani \& Wolf, 2010).

The second subset of transformation models is the interpolation models. The presented research has employed two approaches: finite element method transformation and analytical spline transformation. Unlike the first subset, the 
models of the second subset use the sample of reference points as error-free and draw the interpolation surface exactly through these points. The rest of the reference points are being used for the check.

Finite element method transformation. The key features of using FEM are types of finite elements and partitioning methods. There are many types of 2D finite elements, e.g., triangular, quadrilateral, quadratic triangular, elements with curved edges, etc. However, in geodesy, the most common are simple triangular elements (Figure 2).

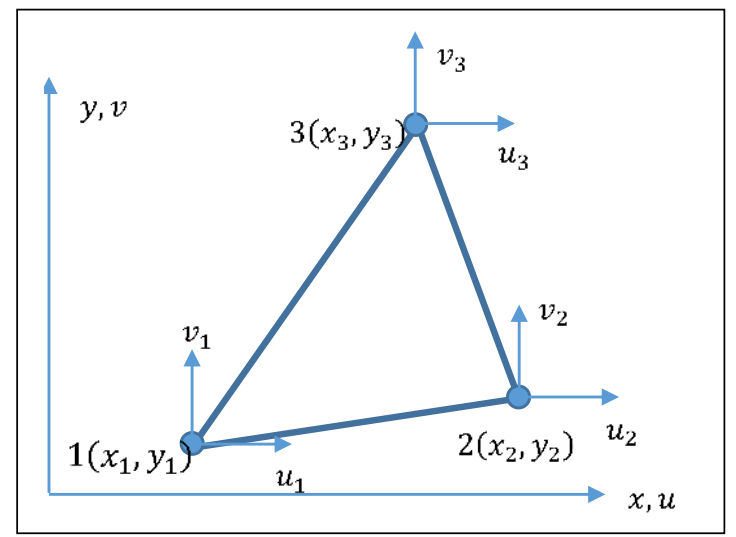

Figure 2. Scheme of triangular planar finite element (Liu \& Quek, 2003)

Among the majority of partitioning methods, geodesists use Delaunay triangulation as an optimal partitioning method based on triangles. The affine transformation (3) is recommended as a transformation function for triangular elements. For each node in the triangular, we know the coordinates in both systems $\left(x_{i}, y_{i}\right)$ and $\left(u_{i}, v_{i}\right)$. Using the system of algebraic equations, one finds six parameters for equations (3). Having these parameters, we may find coordinates for any point inside the triangle.

Analytical spline transformation. The second interpolation method that had been considered is the transformation by the analytical spline. For the case of 2-D interpolation by the analytical spline, it is recommended using the functional that has the following form:

$$
\Phi=\iint_{S}\left[\left(\frac{\partial^{2} \varphi}{\partial x^{2}}\right)^{2}+\left(\frac{\partial^{2} \varphi}{\partial y^{2}}\right)^{2}+2\left(\frac{\partial^{2} \varphi}{\partial x \partial y}\right)^{2}\right] d x d y=\min .
$$

In order to find the function $\varphi$, it is necessary to resolve the system of linear equations:

$$
\tilde{\mathbf{A}} \mathbf{\Lambda}-\mathbf{L}=0,
$$

$$
\text { where } \tilde{\mathbf{A}}=\left[\begin{array}{cccc}
\mathbf{A} & \mathbf{X} & \mathbf{Y} & \mathbf{C} \\
\mathbf{X}^{T} & 0 & 0 & 0 \\
\mathbf{Y}^{T} & 0 & 0 & 0 \\
\mathbf{C}^{T} & 0 & 0 & 0
\end{array}\right], \quad \boldsymbol{\Lambda}=\left[\begin{array}{c}
\lambda_{0} \\
\lambda_{1} \\
\ldots \\
\lambda_{n-1}
\end{array}\right], \mathbf{X}=\left[\begin{array}{c}
x_{0} \\
x_{1} \\
\ldots \\
x_{n-1}
\end{array}\right], \mathbf{Y}=\left[\begin{array}{c}
y_{0} \\
y_{1} \\
\ldots \\
y_{n-1}
\end{array}\right], \mathbf{C}=\left[\begin{array}{c}
1 \\
1 \\
\ldots \\
1
\end{array}\right] .
$$

The system of equations is being split into two separate systems.

$$
\begin{gathered}
\tilde{\mathbf{A}} \boldsymbol{\Lambda}_{x}-\Delta \mathbf{X}=0, \tilde{\mathbf{A}} \mathbf{\Lambda}_{y}-\Delta \mathbf{Y}=0 \\
\Delta \mathbf{X}=\left[\begin{array}{c}
\delta x_{0}=x_{0}-u_{0} \\
\delta x_{1}=x_{1}-u_{1} \\
\cdots \\
\delta x_{n-1}=x_{n-1}-u_{n-1}
\end{array}\right], \Delta \mathbf{Y}=\left[\begin{array}{c}
\delta y_{0}=y_{0}-v_{0} \\
\delta y_{1}=y_{1}-v_{1} \\
\cdots \\
\delta y_{n-1}=y_{n-1}-v_{n-1}
\end{array}\right] .
\end{gathered}
$$

For the matrix $\mathbf{A}$, the coefficients $a_{i j}$ are being calculated in the following manner:

$$
a_{i j}=a_{j i}=r_{i j} \ln r_{i j}, r_{i j}=\left(x_{i}-x_{j}\right)^{2}+\left(y_{i}-y_{j}\right)^{2} \text {. }
$$

After carrying out the calculations, one can find the transformed coordinates of any point using the next statements: 


$$
\begin{aligned}
& u=x+\sum_{i=0}^{n-1} r_{i} \lambda_{x_{i}}+x \lambda_{x_{n}}+y \lambda_{x_{n+1}}+\lambda_{x_{n+2}} ; \\
& v=y+\sum_{i=0}^{n-1} r_{i} \lambda_{y_{i}}+x \lambda_{y_{n}}+y \lambda_{y_{n+1}}+\lambda_{y_{n+2}} .
\end{aligned}
$$

These expressions form the interpolation surface with continuous the first and the second derivatives.

All the considered models were applied for the search of transformation parameters to interconnect the old local CS of Almaty and the new one CS based on UTM.

\section{Results and analysis}

The results and analysis of the transformation parameters determination for the first subset of models are presented below. For the determination of the transformation parameters, 18 points with known coordinates in both CSs were chosen. The research (Ocalan, 2019) provide confirmatory evidence that the number is sufficient for reliable transformation. The primary requirement for the accuracy $m_{x}, m_{y}$ of transformation is that it must be confined to $0.05 \mathrm{~m}$ range for each axis. Statistical significance was accepted at the $99 \%$ level. It means that the probability coefficient $t$ equals 2.5. This criterion has been used to check the transformation quality. The extreme deviations $\Delta_{x}, \Delta_{y}$ after transformation have to be equal or less the values:

$$
\Delta_{x}, \Delta_{y} \leq t m_{x}, t m_{y} .
$$

The data generated by the study of approximation models are reported in Table 1.

Table 1. Transformation quality for approximation models

\begin{tabular}{|l|c|c|c|c|c|c|}
\hline \multicolumn{1}{|c|}{ Model } & $\begin{array}{c}\text { RMS x, } \\
\mathrm{m}\end{array}$ & $\begin{array}{c}\text { RMS y, } \\
\mathrm{m}\end{array}$ & $\begin{array}{c}\text { Max abs } \\
\text { deviation X, } \mathrm{m}\end{array}$ & $\begin{array}{c}\text { Max abs } \\
\text { deviation Y, } \mathrm{m}\end{array}$ & $\Delta_{x}, \mathrm{~m}$ & $\Delta_{y}, \mathrm{~m}$ \\
\hline Helmert transformation & 1.133 & 0.401 & 1.709 & 0.682 & 2.832 & 1.002 \\
\hline Affine transformation & 0.147 & 0.152 & 0.204 & 0.246 & 0.368 & 0.380 \\
\hline Bilinear transformation & 0.266 & 0.095 & 0.484 & 0.165 & 0.665 & 0.238 \\
\hline $2^{\text {nd }}$ order polynomial transformation & 0.061 & 0.074 & 0.125 & 0.119 & 0.152 & 0.185 \\
\hline $3^{\text {rd }}$ order polynomial transformation & 0.056 & 0.051 & 0.114 & 0.096 & 0.140 & 0.128 \\
\hline $4^{\text {th }}$ order conformal polynomial transformation & 0.088 & 0.288 & 0.177 & 0.298 & 0.220 & 0.670 \\
\hline
\end{tabular}

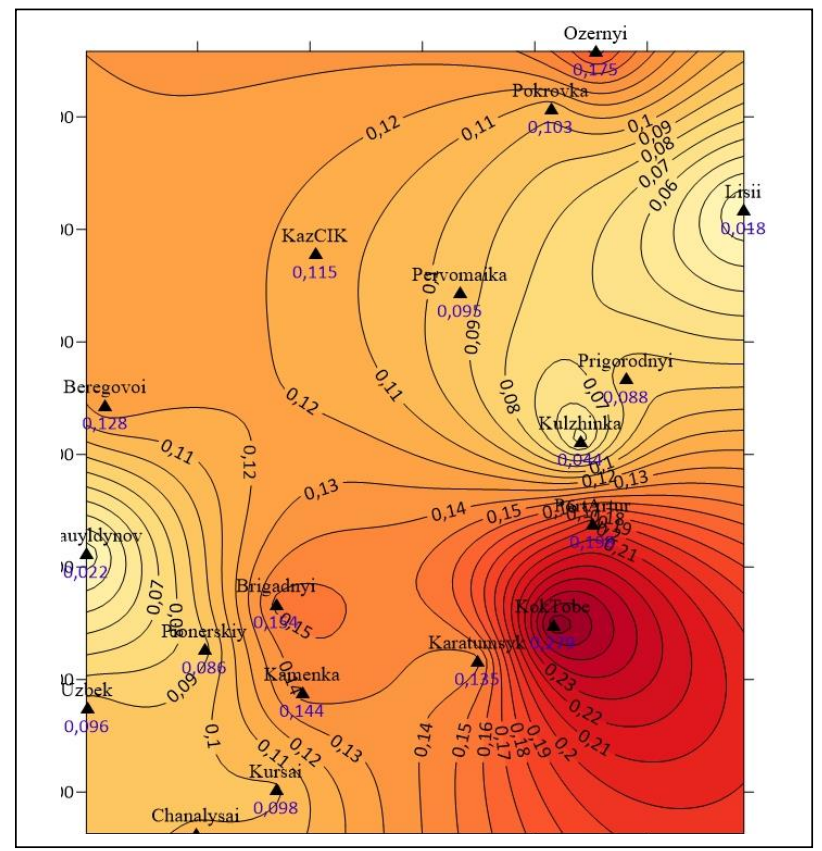

Figure 3. The image of the deformation field for affine transformation 
Extreme deviations fall into range defined by expressions (10). Despite that, the standard deviations do not satisfy the primary requirement. The closer to the primary demand is the third-order polynomial transformation. The results reveal a high probability of gross errors in initial data. To localize these errors were constructed deformation fields that help to analyze transformation results visually (Figure 3).

The visual presentation provides strong evidence that there is at least one point with considerable deformation. Such points have to be excluded from the analysis. However, such an approach may lead to a wrong decision. The considered above polynomial transformations tend to spread the local gross errors to the whole network. That is why we may delete the point that really does not have the gross errors. It is possible to put across the problem using the methods that allow localizing gross errors in particular regions and reduce their influence on the whole network. Among such methods, we have studied FEM transformation and analytical spline transformation.

For the FEM transformation, the network was divided into triangles using Delaney triangulation (Figure 4).

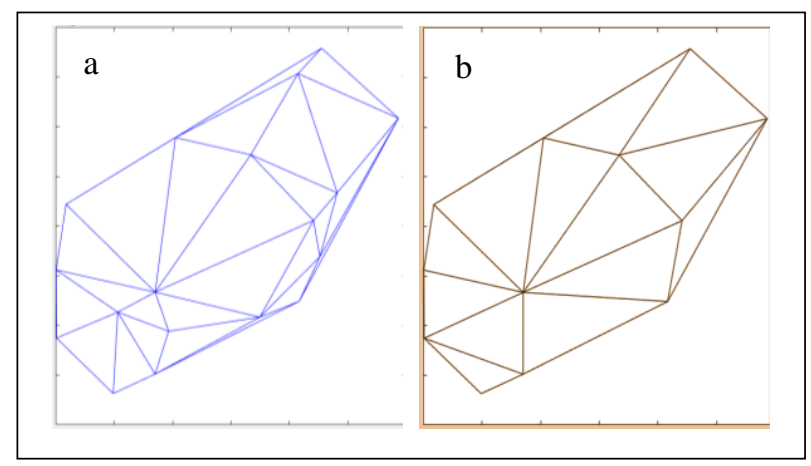

Figure 4. The scheme of Delaunay triangulation for a) the whole network; b) particular network without points that were excluded for checking

For each triangle, the transformation parameters were found using affine transformation with the necessary accuracy. The transformation quality was checked for six points left. The data generated by the study of interpolation models are reported in Table 2.

Table 2. Transformation quality for interpolation models

\begin{tabular}{|l|c|c|c|c|c|c|}
\hline \multicolumn{1}{|c|}{ Model } & RMS x, m & RMS y, m & $\begin{array}{c}\text { Max abs } \\
\text { deviation X, m }\end{array}$ & $\begin{array}{c}\text { Max abs } \\
\text { deviation Y, } \mathrm{m}\end{array}$ & $\Delta_{x}, \mathrm{~m}$ & $\Delta_{y}, \mathrm{~m}$ \\
\hline Analytical spline transformation & 0.030 & 0.270 & 0.033 & 0.300 & 0.075 & 0.675 \\
\hline Finite element method transformation & 0.102 & 0.129 & 0.263 & 0.173 & 0.255 & 0.322 \\
\hline
\end{tabular}

The data yielded some interesting findings. A closer look at the data indicates that the analytical spline transformation provides very accuracy for the transformation along the Y-axis. At the same time, FEM transformation accuracy has an insufficient but uniform accuracy. These figures suggest that both methods, regardless of interpolation models, cannot afford the necessary accuracy of the transformation. But, unlike the approximation models, the interpolation models allow localizing gross errors. By the results of the analysis, the gross error was found for the same point in both models. This point was excluded, and the new transformation parameters search has been carried out. The new results provided convincing evidence showing that both models work well provide sufficient accuracy, namely: for the FEM transformation $m_{x}=0.027 \mathrm{~m}, m_{y}=0.039 \mathrm{~m}$; for the analytical spline transformation $m_{x}=$ $0.018 \mathrm{~m}, m_{y}=0.052 \mathrm{~m}$. For these two methods, the biggest absolute deviation reaches $0.072 \mathrm{~m}$, which is certainly less than the threshold value.

\section{Conclusions}

In this paper, the discussion centers on the transformation parameters determination of nonhomogeneous coordinate systems. The research has been carried out for the renovation and restoration of the coordinate system of Almaty, Kazakhstan. Given the centrality of this issue, in the paper were studied the different approaches for transformation parameters acquisition: Helmert transformation, bilinear transformation, the second and third-order polynomial transformation, and the fourth-order conformal polynomial transformation. An alternative approach was considered the transformation using interpolation models, such as the finite element method and analytical spline transformation. The data gathered in the study suggest that neither of the discussed methods provides the necessary transformation 
accuracy $(>5 \mathrm{~cm})$. The results yielded by this study provide convincing evidence that for the case of nonhomogeneous networks, the foremost task is the identification of gross errors. Since approximation methods redistribute corrections to the points of the whole network, it is suggested using interpolation models for error identification. The underlying argument in favor of interpolation methods is proved by simulation results. There are two main arguments that can be advanced to support the use of interpolation methods. Firstly, these methods describe non-uniform deformations of local coordinate systems. Secondly, these methods help to localize the gross distortions. On the basis of the evidence available, it seems fair to suggest at the first step to use finite element method transformation to identify places with large deformations. Then, it is possible to use either one of the approximation models and determine the transformation key or use refined finite element transformation.

Despite in-depth research, much has to be done. Further research in this area may include a study of different transformation models with their weight matrixes and a comparison of existing models with transformation by neural networks.

\section{References}

Deakin, R. E. (2004). Coordinate transformations in surveying and mapping (Technical Report, pp. 1-31). School of Geospatial Sciences, RMIT University.

Gao, Y. (2017). Analysis of coordinate transformation with different polynomial models (Bachelor thesis). Universität Stuttgart, Stuttgart, Germany. https://elib.uni-stuttgart.de/bitstream/11682/9661/1/BscThesis_GaoYueqing.pdf

Ghilani, C., \& Wolf, P. R. (2010). Adjustment computations: Spatial data analysis (5th ed.). Hoboken.

Gil, J., \& Mrówczyñska, M. (2012). Methods of artificial intelligence used for transforming a system of coordinates. Geodetski list, $4,321-336$.

Kohli, A., \& Jenni, L. (2008, June). Transformation of cadastral data between geodetic reference frames using finite element method. Paper presented at the FIG Working Week 2008, Stockholm, Sweden.

Liu, G. R., \& Quek, S. S. (2003). The finite element method: a practical course. Butterworth-Heinemann.

Mikhail, E. M. (1976). Observations and least squares. IEP - A Dunn-Donnelley Publisher.

Ocalan, T. (2019). Investigation on the effects of number of common points in $2 \mathrm{~d}$ transformation problem. International Journal of Engineering and Geosciences (IJEG), 4(2), 58-62. https://doi.org/10.26833/ijeg.446962

The International Association of Oil \& Gas Producers. (2018). Geomatics Guidance Note 7, part 2. Coordinate Conversions \& Transformations including Formulas (Report No. 373-7-2).

Ziggah, Y. Y., Youjian, H., Tierra, A. R., \& Laari, P. B. (2019). Coordinate transformation between global and local data based on artificial neural network with K-Fold cross-validation in Ghana. Earth Sciences Research Journal, 23(1), 67-77. https://doi.org/10.15446/esrj.v23n1.63860

\section{Notations}

Variables and functions

$a_{0}, a_{1}, a_{2}, \ldots, b_{8}, b_{9}$ - transformation coefficients for models (1)-(6).

\section{Abbreviations}

CS - coordinate system;

FEM - Finite Element Method;

RMS - Root Mean Square Error;

GNSS - Global Navigation Satellite System;

UTM - Universal Transverse Mercator Projection. 